\title{
Utilización de iBook en la Enseñanza de Contenidos de Anatomía en Educación Secundaria
}

\author{
Using iBook in Teaching Anatomy Content in Secundary Education
}

Antonio Baena-Extremera \& Antonio Granero-Gallegos

BAENA-EXTREMERA, A. \& GRANERO-GALLEGOS, A. Utilización de iBook en la enseñanza de contenidos de anatomía en educación secundaria. Int. J. Morphol., 31(2):505-511, 2013.

RESUMEN: Esta investigación tuvo como objetivos, mostrar una propuesta de trabajo de contenidos de anatomía con iBook y valorar la experiencia tanto en los profesores como en los alumnos. La muestra utilizada fue de 375 alumnos de Educación Secundaria Obligatoria (179 hombres y 196 mujeres), con edades comprendidas entre 14 y 15 años $(\mathrm{M}=14.56$; DT $=0,78)$ pertenecientes a cinco centros de enseñanza pública de la provincia de Almería y Granada (España). En relación al profesorado, la muestra fue de 11 docentes de Educación Física (7 hombres y 4 mujeres), con edades comprendidas entre los 27 y 51 años (M=37,57; DT=8,36). Se utilizaron dos cuestionarios, uno para el alumno y otro para los profesores, validados previamente, realizando posteriormente análisis descriptivos. Los resultados muestran que la experiencia del trabajo con ibook es valorada como muy novedosa e interesante; no obstante, los profesores consideran estas herramientas poco aplicables. En relación a la eficacia, los docentes piensan que solamente son algo eficaces mientras que los alumnos creen que son muy eficaces en su aprendizaje. Los principales problemas encontrados son: la propia elaboración del ibook, el coste y la accesibilidad para su uso. Posteriormente, los resultados son discutidos.

PALABRAS CLAVE: Metodología; iBook; Educación Física; TIC; Anatomía humana.

\section{INTRODUCCIÓN}

Sobre las posibilidades de las que dispone el sistema educativo para ser capaz de acoger y utilizar correctamente las novedades tecnológicas y digitales se ha especulado mucho durante los últimos años (Sancho \& Correa, 2010). Estamos inmersos en una sociedad en continuo cambio y muchos de los avances en las tecnologías de la información y la comunicación (TIC) tratan de introducirse en las aulas de educación, sin saber a ciencia cierta si producen beneficios en el aprendizaje de los discentes o no. Parece existir una generalizada opinión de que la utilización de estas innovaciones tecnológicas ayudan a los docentes en el proceso de enseñanza de casi todas las materias; pero diversos autores, como Rodríguez (2010), cuestionan la eficacia o el rendimiento de estas innovaciones en los alumnos suponiendo, en algunos casos, más carga lectiva para ellos.

En general, en la enseñanza de contenidos de Anatomía Humana relacionada con la actividad física en Educación Secundaria, los docentes utilizan CDs interactivos, vídeos demostrativos, presentaciones de diapositivas (tipo power-point o impress) y otros recursos similares. Pero a menudo el profesorado encuentra problemas en la enseñanza de los citados contenidos en población juvenil (12-16 años) debido, principalmente, a la dificultad que comporta su comprensión a estas edades. Cierto es que algunos investigadores y experimentados docentes están publicando trabajos y propuestas de aplicación de alguna nueva metodología de enseñanza o de innovación tecnológica en los que se demuestra que éstas contribuyen a conseguir una mejor asimilación de estos contenidos, en las diversas edades. Así, Pellón et al. (2009) llevaron a cabo una investigación sobre contenidos de anatomía en estudiantes universitarios y pudieron observar que no existía coherencia entre las prácticas y las metodologías planificadas, evidenciando deficiencias en la formación de los alumnos. Otros autores, como Fonseca \& Fonseca (2010), utilizaron como metodología de enseñanza el abordaje de imágenes en estudiantes universitarios para el aprendizaje de Anatomía Patológica, obteniendo resultados muy satisfactorios.

\footnotetext{
* Facultad de Ciencias del Deporte, Universidad de Murcia, Murcia, España.

** Centro del Profesorado Almería 03, Consejería de Educación, Junta de Andalucía, España.
} 
En relación al uso de las TIC y las innovaciones tecnológicas, Ávila et al. (2002) presentaron un trabajo con el uso de las TIC para la enseñanza/aprendizaje de la Biología Celular, Histología y Embriología, llegando a la conclusión de que el uso de estos recursos permitió la integración de actividades en el aula o el uso de la computación e Internet. Otros autores, como Algieri et al. (2009), utilizaron igualmente estas innovaciones en la enseñanza del aparato digestivo, reafirmando la necesidad de integrar en el sistema educativo el uso de las TIC. Por último, Prieto et al. (2011) utilizaron un blog en enseñanza de la placenta comparada; los resultados mostraron que esta metodología, con base en la utilización de blog, fue valorada para el aprendizaje desde el punto de vista clínico, en el autoaprendizaje como material original de apoyo e, incluso, para el público no experto, como novedoso y sorprendente.

Las últimas innovaciones que llegan a las aulas son los ibooks. Se tratan de libros digitales para ser leídos en el ordenador o en una tablet y que además de contener la información por escrito -como los libros en papel-, tienen otras ventajas, ya que pueden incluir fotos interactivas, imágenes en 3D con posibilidad de rotar e, incluso, vídeos. Actualmente apenas existen libros de texto para la enseñanza de contenidos escolares en formato ibook. Por ello, el presente artículo tiene un doble objetivo: 1) Mostrar una propuesta de trabajo de contenidos de anatomía con ibook; 2) Analizar la valoración de la experiencia tanto en profesores como en alumnos.

\section{MATERIAL Y MÉTODO}

Muestra. En este estudio participaron un total de 375 alumnos en clases de Educación Física (EF) pertenecientes al 3er curso de Educación Secundaria Obligatoria (ESO), con edades comprendidas entre 14 y 15 años ( $\mathrm{M=14,56;} \mathrm{DT}=0,78)$. Entre el alumnado, 179 eran hombres y 196 mujeres, pertenecientes a cinco centros de enseñanza pública de las provincias de Almería y Granada (España).

Respeto a los profesores, la muestra fue de 11 profesores de EF (7 hombres y 4 mujeres), con edades comprendidas entre los 27 y 51 años (M=37,57; $\mathrm{DT}=8,36)$, todos profesores funcionarios del cuerpo de Educación Secundaria.

Diseño y procedimiento. Se utilizó un diseño no experimental, descriptivo y transversal. Todos los grupos, recibieron una unidad didáctica de Anatomía del Ejercicio, dentro del área de EF, con una duración de 8 sesiones (un mes de duración aproximadamente). En esta unidad de trabajo la

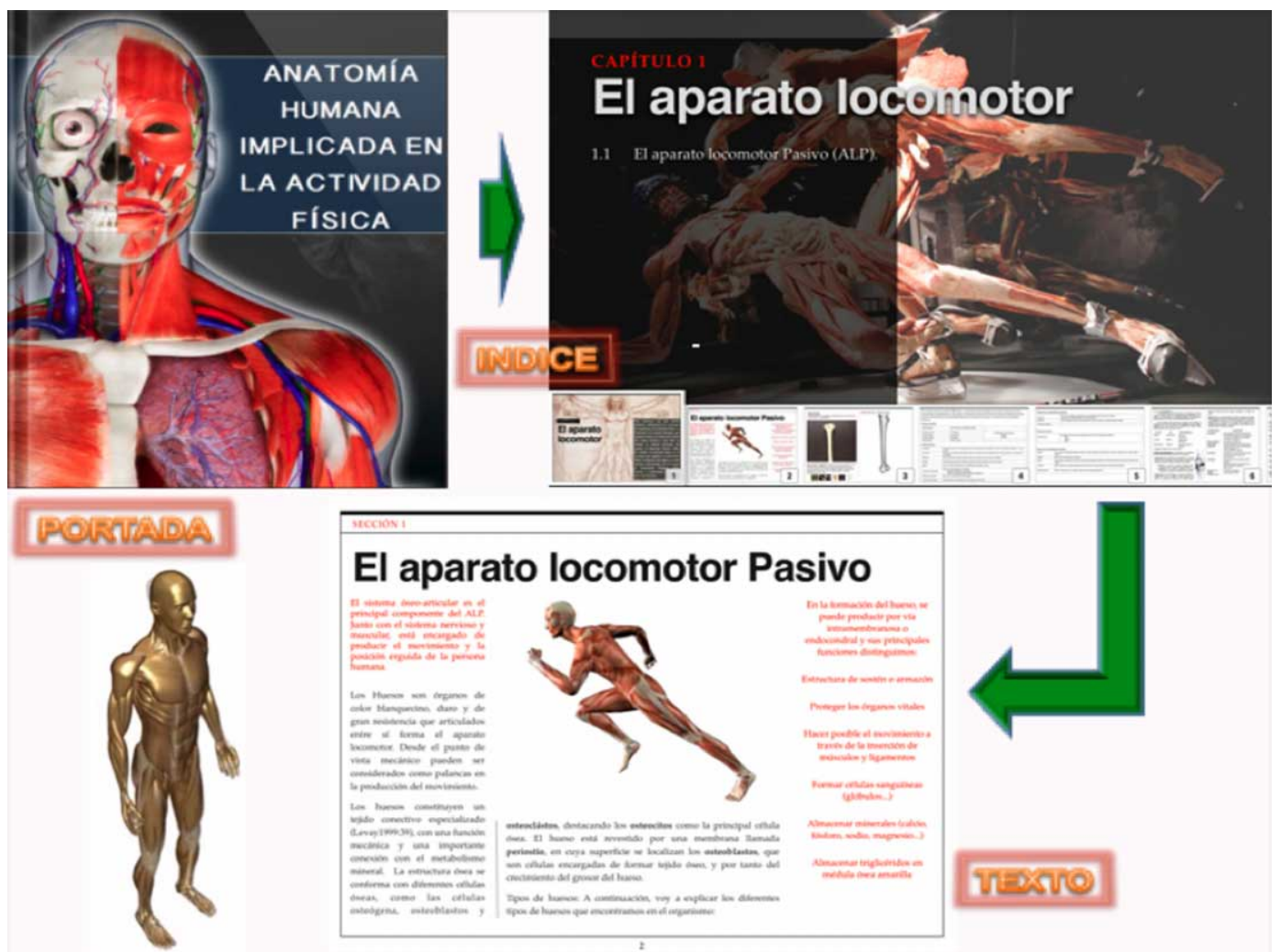

Fig. 1. Portada, índice y parte del texto. 
parte teórica de los contenidos se impartieron a través de la presentación de un ibook con ayuda de un proyector en 2 horas lectivas de clase teórica presencial. A esta parte teórica hay que sumarle una carga extralectiva para los alumnos, en horario de tarde, de 4 horas de trabajo en la biblioteca del centro educativo, donde tenían posibilidad de trabajar en grupos con el ibook, siempre con la presencia del docente de EF.

Para llevar a cabo este trabajo se seleccionaron aquellos centros $(n=5)$ que disponían de los recursos necesarios para que el alumnado pudiera utilizar estas herramientas TIC. El profesorado seleccionado, junto con los investigadores de este trabajo, fueron los encargados de diseñar el ibook en relación a los contenidos de Anatomía Humana implicada en la actividad física que se utilizaría en dicha unidad didáctica para todos los alumnos de los centros participantes en este proyecto. Para la elaboración de los ibooks los profesores mantuvieron varias reuniones previas; primero se seleccionaron los contenidos a trabajar, a continuación se idearon propuestas para el libro digital y, finalmente, se elaboró la herramienta definitiva. Estos ejemplares fueron descargados en los ipads de los docentes y en los propios de cada centro educativo disponibles para las horas de biblioteca. De esta manera, los alumnos podían visionarlos y estudiarlos a su ritmo durante las horas extralectivas. En la Figura 1 se expone un ejemplo de la portada, índice y parte del texto del ibook diseñado. En la Figura 2 se ofrece un ejemplo de imágenes interactivas en $3 \mathrm{D}$, diseñadas y preparadas para que el alumnado interactuara con las mismas durante las sesiones de biblioteca. En la Figura 3 se puede observar cómo también el ibook contenía actividades con posibilidades de evaluación y autoevaluación interactiva por parte del alumnado.

En relación a los contenidos, algunos de los aspectos trabajados son:

- Conocimiento e identificación de los elemento anatómico-funcionales del organismo en relación al ejercicio físico. - Tipo de huesos y articulaciones que participan en los movimientos fundamentales del organismo.

- Funciones de los huesos y articulaciones en el ejercicio físico.

- Estructura micro y macroscópica de huesos y articulaciones.

En las Figuas 1, 2 y 3 se ejemplifica una parte del ibook utilizado.

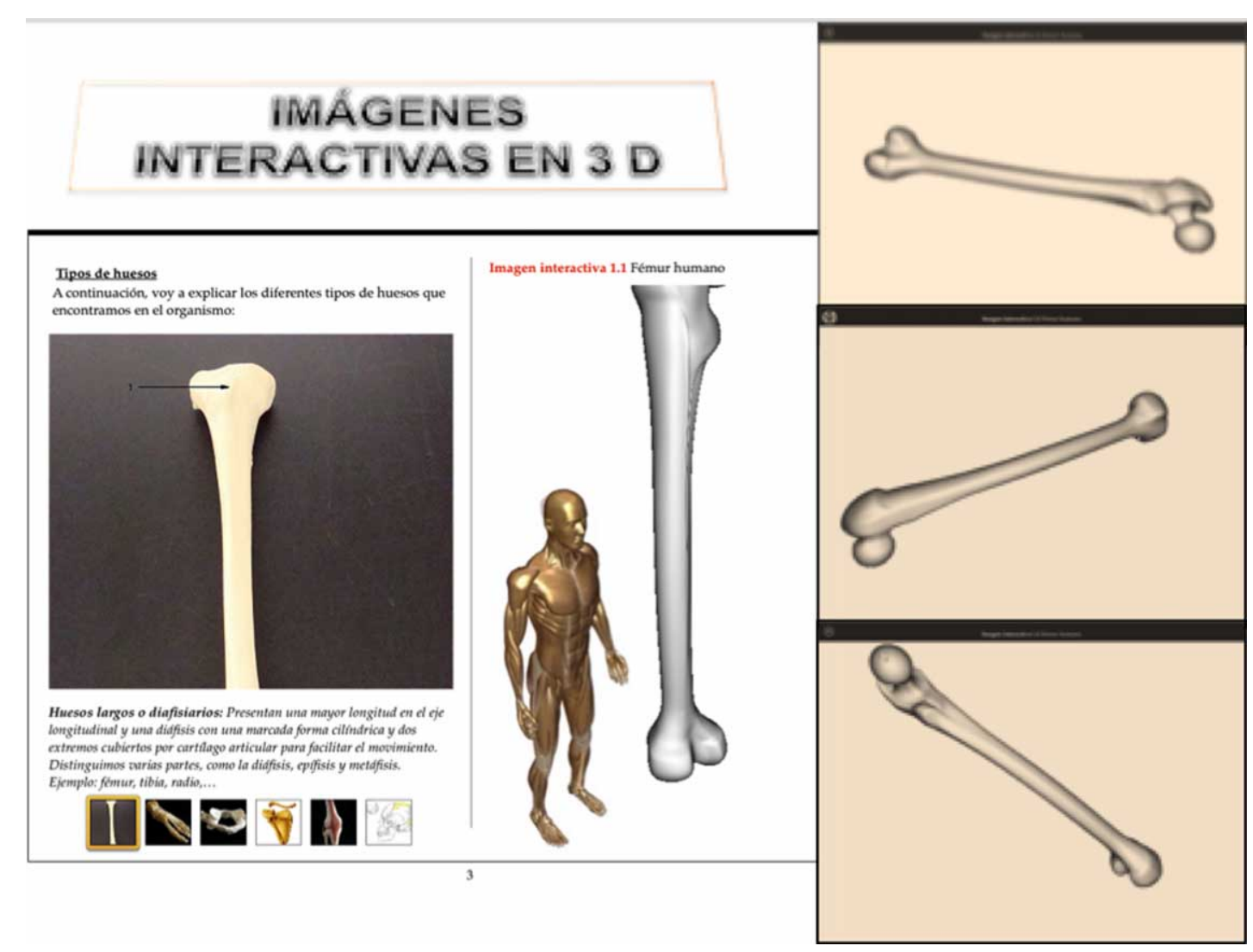

Fig. 2. Posibilidades de interactividad con imágenes 


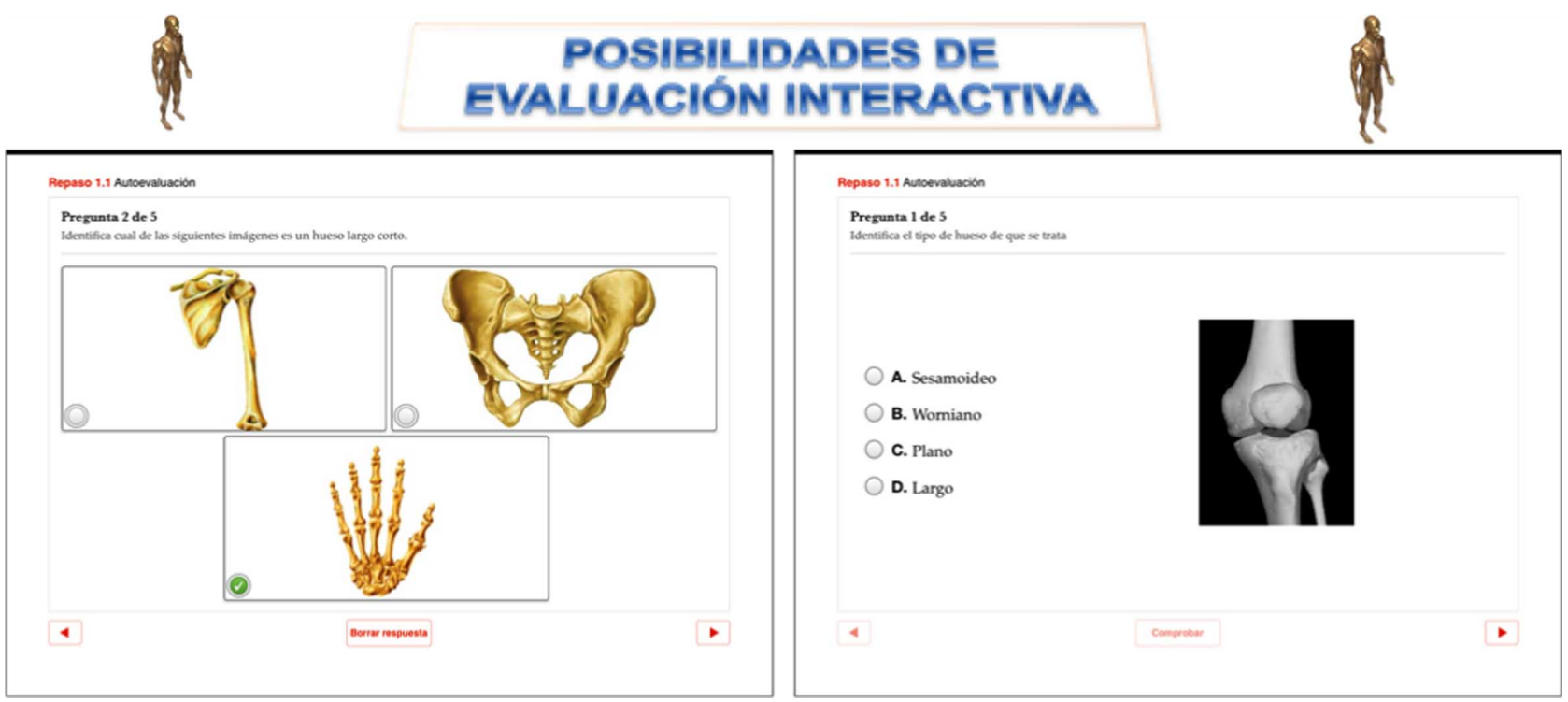

Fig. 3. Posibilidades de realizar evaluaciones interactivas.

Instrumento. El instrumento utilizado para la recopilación de la información fue un cuestionario autoadministrado al final de la unidad didáctica, tanto para el alumnado como para los profesores. El cuestionario de los discentes se compuso de 20 preguntas en relación a la calidad de la actividad y de los contenidos trabajados: novedad, interés, aplicabilidad, eficacia y comprensión, y mejoras o problemáticas con respecto a los libros en papel. El cuestionario de los docentes se componía de 10 preguntas, con especial hincapié en las posibles mejoras, o no, de la utilización de estos libros, novedad, tiempo de trabajo previo en su preparación, eficacia en el proceso de enseñanza, viabilidad práctica, etc. Para la validación de los instrumentos de medida se llevaron a cabo los siguientes procedimientos: primero, con el objetivo de comprobar si el contenido de las preguntas, terminología y vocabulario eran los adecuados, tres expertos (uno en educación, otro en anatomía y un tercero en creación de materiales didácticos) valoraron el mismo, aportando posibles modificaciones y mejoras. Seguidamente, se procedió al examen y validación del cuestionario por tres expertos en esta metodología de investigación, realizando las modificaciones aportadas.

Análisis estadístico. Tras la codificación y tabulación de los datos, se realizó un análisis descriptivo de las frecuencias con SPSS v.17.

\section{RESULTADOS Y DISCUSIÓN}

La primera cuestión iba referida a la novedad en el modelo de enseñanza de los aspectos teóricos sobre los contenidos de Anatomía del Ejercicio propuestos en la unidad didáctica (explicación teórica y posterior estudio de los contenidos con el ibook). Los resultados se puede observar en la Figura 4.

Prácticamente la totalidad de la muestra consideró la utilización del ibook como una técnica novedosa y muy interesante en la presentación de los contenidos teóricos de Anatomía del Ejercicio, tanto alumnos $(81 \%$ y $92 \%$, respectivamente) como profesores $(69 \%$ y $80 \%$, respectivamente). Esto pone de manifiesto la adecuación de estas herramientas a los intereses y motivaciones, sobre todo del alumnado. Estos datos suponen el planteamiento de algunas reflexiones, siguiendo a Balanskat et al. (2006), Cox \& Abbott (2004a, 2004b), Kozman (2003) y Sancho (2006), respecto a que las mejoras en el aprendizaje de los alumnos se deban al uso pedagógicamente innovador de las TIC. No obstante, habría que demostrar si ocurre igual en otros contenidos. Por ello, el aprendizaje puede deberse a que esta metodología les resulte novedosa e interesante y no tanto a factores relacionados con la eficacia de estas herramientas.

Otra de las preguntas a destacar en esta investigación iba referida a las posibilidades de aplicación de los ibook en clase, de manera que profesorado y alumnado debían determinar en una escala el grado y posibilidades de aplicación de estas herramientas en el aprendizaje de contenidos de este tipo (Fig. 5).

Como se puede observar, la gran mayoría de alumnos $(76 \%)$, por una menor proporción entre el profesorado, 
opinaron muy favorablemente sobre las posibilidades de aplicación de estas herramientas en los contenidos de Anatomía del Ejercicio. Asimismo, es destacable, como se puede apreciar en la Figura 5, que los docentes vean una mayor dificultad en la aplicación de estos libros, habiendo incluso un $13 \%$ del profesorado que opina que es poco aplicable para estos contenidos. Numerosas investigaciones (Pelgum, 2001; Conlon \& Simpson, 2003) han puesto de manifiesto que uno de los principales obstáculos para aplicar las TIC son la organización y la cultura tradicional a la que la escuela está sometida (Alonso et al., 2010), sobre todo, en áreas de conocimiento como la que estamos tratando. A pesar de ello, aunque la mayoría de los centros educativos de los países tecnológicamente desarrollados tienen acceso a ordenadores e Internet, sólo un reducido número de docentes los utiliza (Sigalés et al., 2007; Law et al., 2008), y este número es aún más reducido si nos referimos a contenidos de Anatomía del Ejercicio. Esto conlleva, y así queda también reflejado en estos resulta-

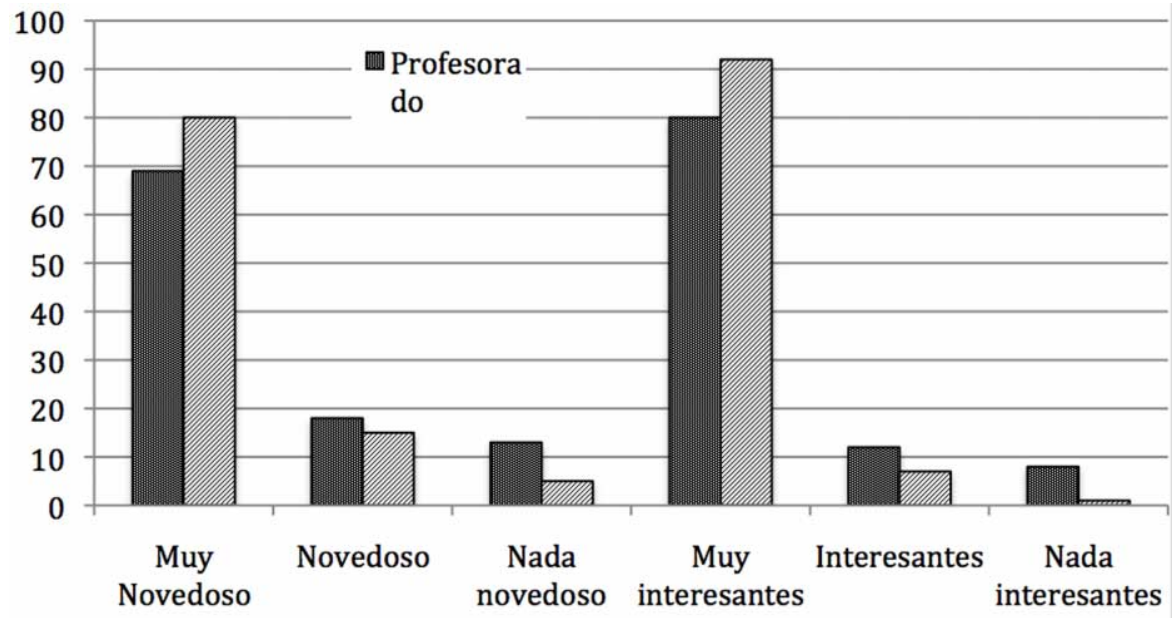

Fig. 4. Novedad e interés en la presentación de los aspectos teóricos.

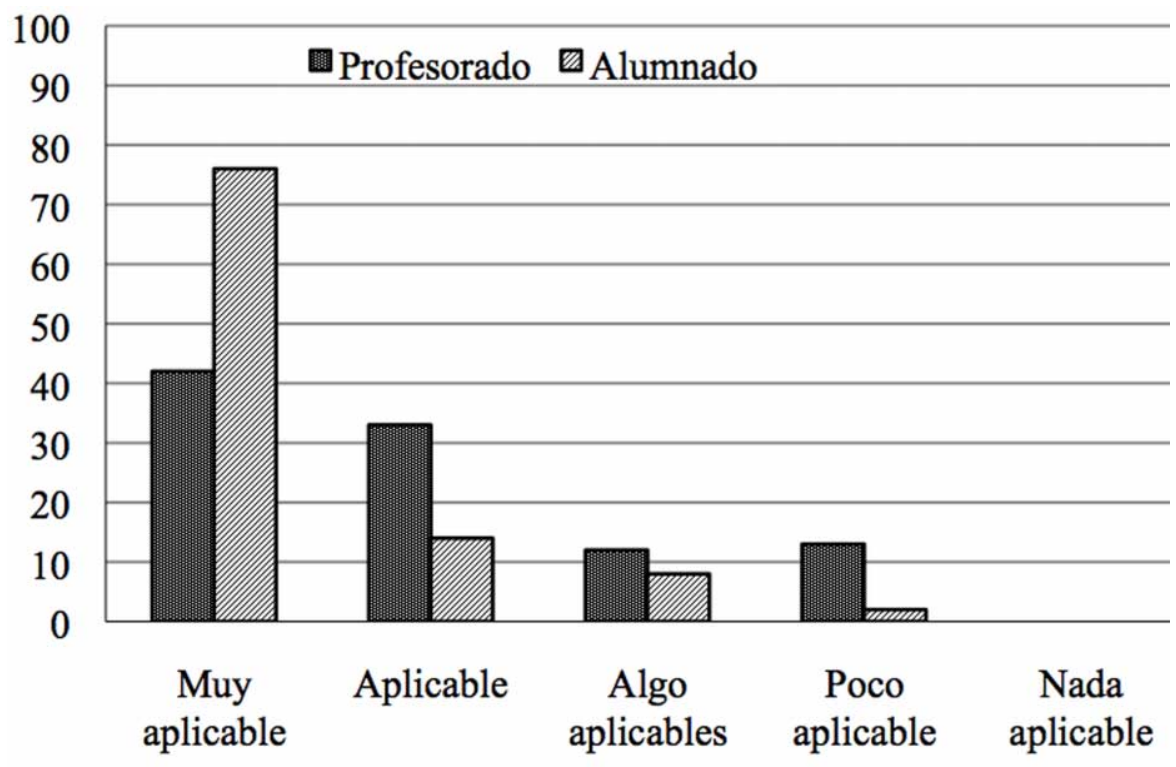

Fig. 5. Posibilidades de aplicación del ibook. dos, que los docentes que usan las TIC tienen dificultades para modificar sus prácticas docentes (McClintock, 2000), dificultando, en parte, la aplicabilidad de estos recursos a la hora de diseñar las clases teóricas, ya que supone un trabajo extra. En cambio, la perspectiva del alumno, como se demuestra, es muy diferente.

La siguiente pregunta de interés para esta investigación (Fig. 6) estaba referida a la eficacia en el proceso de enseñanza (para el docente) y de aprendizaje (para el alumno). Se les pidió que expresaran su opinión sobre el grado de eficacia que demostró esta herramienta en la enseñanzaaprendizaje de los contenidos de anatomía del ejercicio, frente a los libros tradicionales.

Tal como se puede apreciar en la Figura 6, los alumnos creen más que los docentes en la eficacia de estos recursos. Destaca la proporción de alumnos que opinan que es muy eficaz (45\%). Por el contrario, los profesores optan, principalmente, por manifestar que son algo eficaces (39\%), habiendo incluso algunos docentes que opinan que son poco (19\%) o nada eficaces (1\%). Esto posiblemente sea debido a que los docentes aún no creen claramente en la aplicación real de estas innovaciones, pues al ser un recurso tan novedoso, la mayoría de los centros educativos aún no disponen de ibooks. Autores como Majó \& Marqués (2002), preconizan que el uso de las TIC en el proceso de enseñanza y aprendizaje constituye el mejor instrumento para ofrecer una formación adecuada a los alumnos, haciendo por tanto el proceso más eficaz. Sin embargo, nuestros resultados no demuestran una eficacia tan clara, y corroboran más las aportaciones de Balanskat $e t$ 
al., Law et al. y Sigalés et al., quienes consideran que el uso de las TIC no produce mejoras tan claras en la eficacia de las situaciones de aprendizaje en el contexto escolar.

La última cuestión iba dirigida a los profesores y se les preguntaba sobre los principales problemas encontrados a la hora de trabajar con el ibook. Estos docentes, afirmaron (Fig. 7) que el principal problema residía en la elaboración de los libros digitales (34\%). Probablemente ello es debido a que el proceso de edición del mismo resultó complejo y costoso, desde el punto de vista del tiempo que conllevó para el profesorado, y fue necesario la utilización de programas de diseño que, por lo general, los docentes no aprenden en su formación inicial o permanente. El segundo problema destacado en la utilización de estas herramientas era el coste económico (32\%). Esta razón es debida fundamentalmente a que todavía los centros educativos y alumnos no disponen de los recursos económicos suficientes para que todo el alumnado pueda hacer uso de esta tecnología. De ahí que autores como De Pablos et al. (2010) manifiesten que entre los factores facilitadores del uso de las TIC en educación debe estar la aplicación de una políticas educativas ligada a las TIC, en las cuales prioricen la dotación de medios tecnológicos a los centros educativos y fomenten la formación del profesorado, la elaboración de materiales educativos, etc.

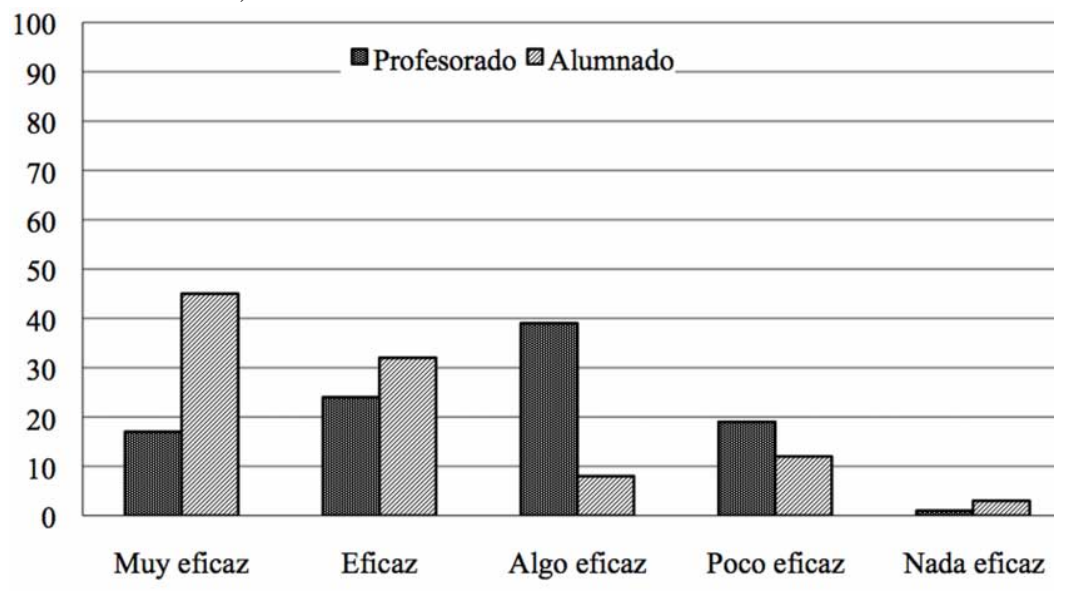

Fig. 6. Eficacia en la enseñanza (del profesor) y aprendizaje (del alumno) con la utilización del ibook.

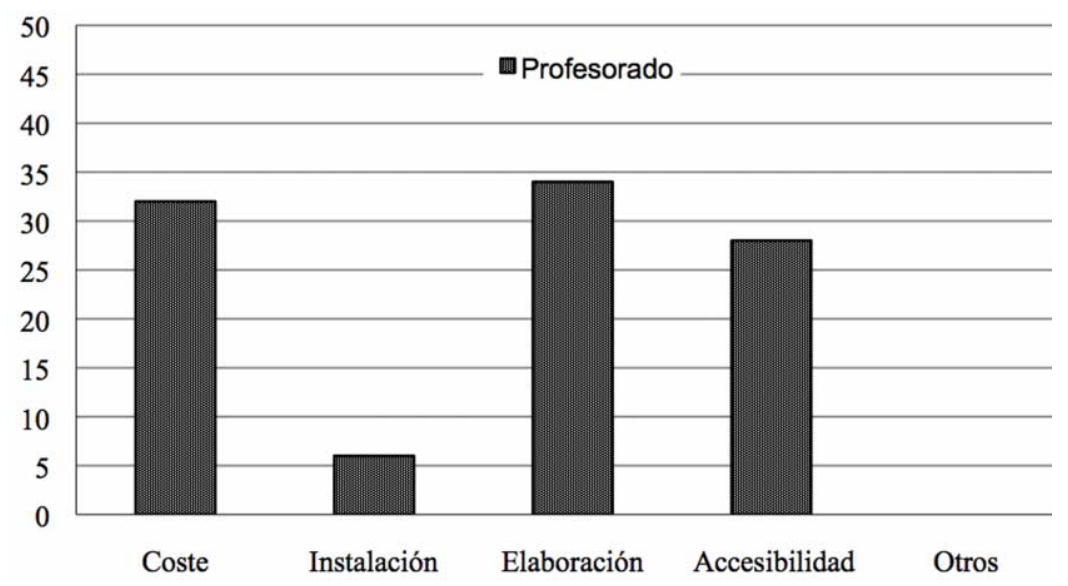

Fig. 7. Principales problemas en su utilización
El tercer problema destacado por los profesores fue la accesibilidad a los alumnos (28\%), pues creen que muchos de ellos tendrán problemas para trabajar con ibooks, dado que en la actualidad aún hay un elevado número familias, alumnos y profesores, que no disponen de la tecnología suficiente como para poder utilizarlo. Esto, obviamente, no es condicionante de que en el futuro se use o sean los libros utilizados pero, en la actualidad, la realidad es otra. En España hay que destacar que para subsanar situaciones de este tipo se ha desarrollado el plan Escuela TIC 2.0, en el que además de elaborar planes de formación TIC para todos los colectivos involucrados, también se financia la adquisición de equipos portátiles para docentes y estudiantes (De Pablos et al.).

Una vez expuesto lo anterior, es necesario concluir afirmando que los ibooks constituyen en la actualidad una herramienta novedosa e interesante, tanto para los profesores como para los alumnos. Pero, no obstante, la aplicación y eficacia de estos libros está aún por determinar. Por ello, creemos que sería necesario realizar más trabajos sobre la eficacia y utilización de ibooks en diferentes muestras de estudiantes, realizar investigaciones pretest-postest e, incluso, comprobar en grupos experimentales si se corroboran o no estos resultados.

BAENA-EXTREMERA, A. \& GRANEROGALLEGOS, A. Using iBook in teaching anatomy content in secundary education. Int. J. Morphol., 31(2):505-511, 2013.

SUMMARY: This study aims, to show a work proposal content iBook anatomy and assess the experience of both teachers and the students. The sample consisted of 375 students of secondary school (179 boys and 196 girls) aged between 14 and 15 years $(M=14.56$, SD $=0.78$ ) from five public schools in the province of Almeria and Granada (Spain). In relation to teachers, the sample was 11 physical education teachers ( 7 men and 4 women), aged between 27 and 51 years $(M=37.57, S D=8.36)$. Two questionnaires were used, one for the student and one for teachers, previously validated and subsequently to descriptive analyzes. The results show that the experience of working with ibook 
is valued as highly novel and interesting, however, teachers consider these tools very applicable. Regarding efficacy, teachers think that they are only somewhat effective while students think they are very effective in their learning. The main concerns are: the proper development of the ibook, cost and accessibility for use. Subsequently, the results are discussed.

KEY WORDS: Methodology; iBook; Physical Education; ICT; Human anatomy.

\section{REFERENCIAS BIBLIOGRÁFICAS}

Algieri, R. D.; Mazzoglio y Nabar, M. J.; Dogliotti, C. G. \& Gazzotti, A. TICs aplicadas a la enseñanza del aparato digestivo. Int. J. Morphol., 27(4):1261-8, 2009.

Alonso, C.; Casablancas, S.; Domingo, L.; Guitert, M.; Moltó, O.; Sánchez, J.A.; et al. De las propuestas de la Administración a las prácticas del aula. Rev. Educ., 352:53-76, 2010.

Avila, R. E.; Samar, M. E.; Chiesa, P.; Camps, D.; Salica, J. P \& Yaryura, G. El uso de las nuevas tecnologías de información y comunicación (NTIC) para la enseñanzalaprendizaje de Biología Celular, Histología y Embriología. Inform. J., 1(1), 2002. Disponible en: http://www.informedicajournal.org/a1n2/ files/papers_informedica/avilal.pdf

Balanskat, A.; Blamire, R. \& Kefala, S. The ICT Impact Report. A review of studies of ICT impact on schools in Europe. 2006. Disponible en: http://ec.europa.eu/education/doc/reports/doc/ ictimpact.pdf

Conlon, T. \& Simpson, M. Silicon Valley verses Silicon Glen: the impact of computers upon teaching and learning: a comparative study. Br. J. Educ. Tech., 34(2):137-50, 2003.

Cox, M. \& Abbott, C. A review of the research literature relating to ICT and attainment. Londres, BECTA. 2004a. Disponible en : http://www.becta.org.uk

Cox, M. \& Abbott, C. An investigation of the research evidence relating to ICT pedagogy. Londres, BECTA. 2004b. Disponible en: http://www.becta.org.uk

De Pablos, J.; Colás, P. \& González, T. Factores facilitadores de la innovación con TIC en los centros escolares. Un análisis comparativo entre diferentes políticas educativas autonómicas. Rev. Educ., 352:23-51, 2010.

Fonseca, G. M. \& Fonseca, A. C. Strategies of Education for the Study of Images in Pathology, School of Dentistry, Universidad Nacional de Cordoba, Argentina. Int. J. Morphol., 28(4):1113-23, 2010.

Kozman, R. B. Technology, Innovation, and Educational Change - A Global Perspective. Washington D.C., ISTE, 2003.
Law, N.; Pelgrum, W. J. \& Plomp, T. Pedagogy and ICT use in schools around the world: Findings from the IEA SITES 2006 study. Hong Kong, CERC-Springer, 2008.

Majó, J. \& Marquès, P. La revolución educativa en la era internet. Barcelona, CissPraxis, 2002.

McClintock, R. Prácticas pedagógicas emergentes. Cuad. Pedagog., 290:74-6, 2000.

Pelgum, W. J. Obstacles to the integration of ICT in education: results from a worldwide educational assessment. Сотр. \& Educ., 37:163-87, 2001.

Pellón, A. M.; Mansilla, S. J. \& San Martín, C. D. Challenges to the Didactic Transposition and Knowledge of the Content in Teaching of Anatomy: Obstacles and Projections. Int. J. Morphol., 27(3):743-50, 2009.

Prieto, R.; Smok, C. \& Rojas, M. Blog Experiences: Compared Placenta. Int. J. Morphol., 29(2):432-5, 2011.

Rodríguez, R. S. El impacto de las TIC en la transformación de la enseñanza universitaria: repensar los modelos de enseñanza y aprendizaje. Teor. Educ., 11(1):32-68, 2010.

Sancho, J. M. De tecnologías de la información y la comunicación a recursos educativos. En: Sancho, J. M. Tecnologías para transformar la educación. Madrid, AKAL/UNIA, 2006. pp.1536.

Sancho, J. M. \& Correa, J. M. Cambio y continuidad en sistemas educativos en transformación. Rev. Educ., 352:17-21, 2010.

Sigalés, C.; Mominó, J. M., \& Meneses, J. L'escola a la Societat Xarxa: Internet a l'educació Primària i Secundària. Informe final de recerca. Universitat Oberta de Catalunya, 2007. Disponible en: http://www.uoc.edu/in3/pic/cat/escola_xarxa/ informe.html

\section{Dirección para Correspondencia: \\ Dr. Antonio Baena-Extremera \\ Facultad de Ciencias del Deporte \\ Universidad de Murcia \\ C/ Argentina s/n 30720 \\ Santiago de la Ribera, Murcia \\ ESPAÑA}

Email: abaenaextrem@um.es

Recibido : 12-03-2012

Aceptado: 27-02-2013 